\title{
Essai de Barymétrie chez le Dromadaire nord-africain
}

\author{
par $A$. BOUÉ, vétérinaire lieutenant
}

\section{DE LA RELATION ENTRE IE POIDS ET LA MASSE}

LE tractus digestif du dromadaire possède une capacité moyenne qui est sensiblement égale à celle du boeuf. Mais la physiologie un peu spéciale du chameau permet et facilite grandement d'énormes différences de régime telles qu'on ne saurait en observer chez le bœuf. La nature du pâturage sous ses multiples aspects, le rythme de l'abreuvement souvent rompu, le régime auquel l'animal est soumis et le travail qu'il accomplit, sont autant de facteurs interférents qui engendrent de nombreuses et brutales variations à grande amplitude du volume alimentaire. pouvant aller en très peu de temps de la vacuité à la réplétion des réservoirs digestifs. C'est ainsi qu'un animal ayant jeuné 48 heures absorbera le troisième jour 120 à 150 kilos de fourrage vert (Vallon). Il pompe 40 litres d'eau s'il est abreuvé tous les trois jours (Cauvet). L'explorateur Ismail ben Derba a vu, en août 1858 dans le Sud Algérien, un de ses chameaux boire exactement 104 litres après être resté plusieurs jours privé d'eau. On peut donc enregistrer pour une même masse anatomique des poids fort différents. Ceci nous a amené à considérer chez le chameau deux poids vifs : le poids net et le poids brut.

Le poids net étant par définition le poids propre d'un corps est le poids de la masse de l'animal plus, évidemment, un certain lest alimentaire; on ne peut obtenir, même par la purgation, la complète vacuité des viscères. On peut avoir ce poids net en laissant l'animal 48 heures à la diète.

Le poids brut est le poids net plus la masse alimentaire que le chameau retient. Ce poids est subordonné aux diverses contingences quotidiennes. donc variable.

Ayant eu la bonne fortune de pouvoir disposer d'un pont-bascule, nous avons essayé d'établir la relation existant entre quelques mensurations et le poids, le poids net s'entend.

\section{DE DIVERSES MENSURATIONS}

Les trois mensurations que nous envisageons ont un rapport étroit et proportionnel à la masse. Ce sont : le périmetre thoracique, le périmètre abdominal et la hauteur.
Le périmètre thoracique est conditionné par le développement de l'animal, par son squelette; le thorax est étroitement lié à la charpente osseuse, il est par excellence la région utile puisqu'il supporte la charge par le bât ou la selle.

Le périmetre abdominal au niveau de la bosse est fonction de l'état d'engraissement. Une bosse bien replète traduisant un bon embonpoint augmentera cette mesure et inversement.

La hauteur renseigne sur le modèle.

D'autres mensurations telles que le périmètre du métacarpien, la longueur du corps, de l'avantbras, du cou, de la tête ne nous ont pas apparu comme ayant toujours un rapport constant avec le poids.

Comment prendre ces mensurations? L'animal étant à la diète depuis 48 heures, les mesures sont prises avec une ficelle ou un ruban étalonnés que l'on passe autour du corps de l'animal debout (1). Le perimètre thoracique est pris derrière la callosité sternale, la périmètre abdominal à l'endroit le plus haut de la bosse, en son milieu, mesure prise à lá fin de l'expiration. Pour avoir la taille, une toise même de fortune est fort utile; la hauteur est prise à l'aplomb normal du membre antérieur, la toise est placée à la hauteur de la pointe du coude au milieu de l'avant-bras.

\section{DU RAPPORT POIDS-MENSURATIONS}

Nous avons d'abord cherché la relation qui liait le produit de nos trois mensurations au poids donné par le pont-bascule, puis nous en avons vérifié la valeur pour un usage pratique.

La relation a été établie à partir de méhara du Goum d'Abadla; ceux des Goums de Kenadza, Taghit et Colomb-Béchar ont permis d'en contrôler la véracité. Les chameaux militaires sont dociles et plus faciles à peser. Ils provenaient en grande partie de la région, ceux ayant de la taille ( $1 \mathrm{~m} .80$ minimum) avaient du sang Ouled Sidi Cheikh ou Chambi d'El Goléa; les autres avaient plus le modèle de l'animal de bât que celui du méhari.

(1) Le perimètre abdominal varie selon la station debout ou baraquée de l'animal. 
TABLEAU II

\begin{tabular}{|c|c|c|c|c|c|c|c|c|c|c|}
\hline 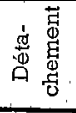 & 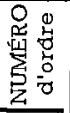 & $\begin{array}{c}\text { No MATRIC. } \\
\text { des } \\
\text { méharistes }\end{array}$ & SEXE & $A G E$ & HAUTEUR & $\begin{array}{c}\text { PÉRIMÈTRE } \\
\text { thoracique }\end{array}$ & $\begin{array}{l}\text { PÉRIMËTRE } \\
\text { abdominal }\end{array}$ & $\begin{array}{c}\text { POIDS } \\
\text { au } \\
\text { pont-bascule }\end{array}$ & $\begin{array}{c}\text { POIDS } \\
\text { nat la relation } \\
\mathrm{P}=53 \mathrm{~T} A \mathrm{H}\end{array}$ & $\begin{array}{l}\text { APPROXI- } \\
\text { MATION }\end{array}$ \\
\hline (1) & (2) & 3. & (4) & (5) & (6) & (7) & (8) & $(9)$ & (10) & (11) \\
\hline \multirow{9}{*}{ 岕 } & 14 & 24 & $\mathrm{H}$ & 12 , & $I, 80$ & 1,98 & 2,23 & 420 & 421,22 & $+1,22$ \\
\hline & 15 & 303 & E & 5 & 1,75 & 1,89 & 2,06 & 377 & 361,11 & $-15,89$ \\
\hline & 16 & 550 & $E$ & 5 & 1,82 & 2,00 & 2,22 & 439 & 428,28 & $-10,62$ \\
\hline & 17 & 283 & $\mathrm{H}$ & 7 & 1,75 & 1,87 & 2,13 & 357 & 369,43 & $+12,43$ \\
\hline & 18 & 291 & $E$ & 10 & 1,84 & 2,00 & 2,18 & 445 & 425,18 & $-19,82$ \\
\hline & 19 & 1039 & E & 8 & 1,79 & 1,85 & 2,02 & 347 & 354,52 & $+7,42$ \\
\hline & 20. & 479 & $\mathrm{H}$ & 10 & 1,79 & 2,00 & 2,22 & 420 & 421,22 & $+1,22$ \\
\hline & 21 & 456 & $\mathrm{H}$ & 6 & 1,81 & 1,95 & 2,22 & 400 & 415,28 & $+15,28$ \\
\hline & 22 & 264 & $\mathrm{H}$ & 7 & $1^{\prime}, 76$ & 1,96 & 2,23 & 470 & 407,70 & $-2,30$ \\
\hline \multirow{12}{*}{ 悹 } & 23 & 359 & $E$ & 6 & 1,81 & 1,97 & 2,29 & 450 & 32,76 & $-17,24$ \\
\hline & 24 & 167 & $E$ & 4 & 1,86 & 1,98 & 1,76 & 310 & 343,52 & $+33,52$ \\
\hline & 25 & 467 & $E$ & 14 & 1,75 & 2,02 & 2,32 & 430 & 435,66 & $+4,66$ \\
\hline & 26 & 487 & $E$ & 4 & 1,79 & 1,86 & 2,10 & 330 & 370,55 & $+40,55$ \\
\hline & 27 & 494 & $\mathrm{H}$ & 11 & 1,86 & 2,00 & 2,25 & 450 & 443,61 & - 6,39 \\
\hline & 28 & 497 & $\mathrm{H}^{\prime}$ & 6 & 1,76 & 1,92 & 2,17 & 400 & 388,63 & $-11,37$ \\
\hline & 29 & 522 & $\mathrm{H}^{\prime}$ & 8 & $1 ; 77$ & 2,03 & 2,20 & 410 & 418,95 & $+8,95$ \\
\hline & 30 & 552 & $\mathrm{H}$ & 12 & 1,78 & 1,98 & 2,27 & 420 & 424,02 & $+4,02$ \\
\hline & $3 I$ & 1378 & $H$ & 7 & 1,80 & 1,95 & 2,17 & 410 & 403,68 & $-6,32$ \\
\hline & 32 & 1476 & $\mathrm{H}$ & 6 & $1,87$. & 2,14 & 2,44 & 510 & 517,51 & $+7,51$ \\
\hline & 33 & 500 & $E$ & 5 & 1,69 & 1,76 & 1,92 & 310 & 302,67 & $\rightarrow 7,33$ \\
\hline & 34 & 501 & $E$ & 3 & 1,65 & 1,76 & 1,98 & 290 & 304,74 & $+14,74$ \\
\hline \multirow{14}{*}{ 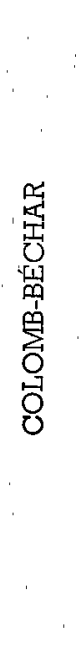 } & 35 & 64 & E & 4 & 1,84 . & 1,98 & 2,12 & 390 & 409,34 & $+19,34$ \\
\hline & 36 & 33 & $E$ & 4 & 1,91 & 2,03 & 2,12 & 455 & 435,65 & $-19,35$ \\
\hline & 37 & 27 & H & 8 & 1,81 & 2,04 & 2,34 & 450 & 457,93 & $+7,93$ \\
\hline & 38 & 54 & $\mathrm{H}$ & 6 & 1,77 & 2,04 & 2,27 & 455 & 434,41 & $-20,59$ \\
\hline & 39 & 131 & $\mathrm{H}$ & 8 & 1,75 & 2,03 & 2,27 & 455 & 427,40 & $-27,60$ \\
\hline & 40 & 132 & $E$ & .5 & 1,82 & 1,98 & 2,27 & 440 & 433,54 & $一 \quad 6,96$ \\
\hline & 41 & 1247 & $\mathrm{H}$ & 7 & 1,69 & 1,85 & 2,20 & 350 & 364,54 & $+4,54$ \\
\hline & 42 & 428 & $\mathrm{H}$ & 9 & 1,79 & 2,05 & 2,47 & 480 & 480,37 & 0,37 \\
\hline & 43 & 824 & $\mathrm{H}$ & 8 & 1,90 & 2,07 & 2,51 & 545 & 523,20 & $\div 21,80$ \\
\hline & 44 & 1294 & $\mathrm{H}$ & 9 & 1,75 & 1,96 & 2,23 & 410 & 405,39 & $-4,61$ \\
\hline & 45 & 92 & $\mathrm{H}$ & 4 & $1 ; 71$ & 1,86 & 2,11 & 380 & 355,68 . & $\therefore 24,32$ \\
\hline & 46 & 107 & $\mathrm{H}$ & 4 & 1,78 & 2,01 & 2,20 & 400 & 418,17 & $i+17,17$ \\
\hline & 47 & 104 & $\mathrm{H}$ & 6 & 1,76 & 1,98 & 2,24 & 400 & 413,71 & $+13,71$ \\
\hline & 48 & 1258 & $E$ & 6 & $1 ; 87$ & 1,94 & 2,08 & 410 & 399,92 & $-10,08$ \\
\hline \multirow{3}{*}{$\sum_{0}^{2}$} & 49 & & $\mathrm{H}$ & 4 & $1,8 \mathrm{I}$ & 1,93 & 2,13 & 405 & 394,35 & $-10,65$ \\
\hline & 50 & . & H & 10 & 1,80 & 2,09 & 2,30 & 443 & 458,58 & $+15,58$ \\
\hline & 51 & & $E$ & 5 & 1,86 & 1,85 & 1,97 & 370 & 359,27 & 10,73 \\
\hline
\end{tabular}


Expérience donnant la relation. - Elle a porte sur 13 animaux du Goum d'Ábadla qui, après une diète de 48 heures, ont été pesés et mesurés (26 janvier 1945). Les résultats sont notés dans le tableau ci-dessous; le poids est exprimé en kilogrammes et les mesures en mètres. Dans la colonne 11, on trouve le rapport : Poids

périmètre thoracique $(\mathrm{T}) \times$ périmètre abdominal $(\mathrm{A}) \times$ hauteur $(\mathrm{H})$ D'après les chiffres de la colonne 11, il ressort que la relation moyenne est de 53 .

TABLEAU I

\begin{tabular}{|c|c|c|c|c|c|c|c|c|c|c|}
\hline 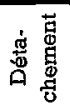 & 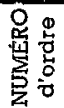 & $\begin{array}{c}\text { No MATRIC. } \\
\text { des } \\
\text { méharistes }\end{array}$ & SEXE & $A G E$ & HAUTEUR & $\begin{array}{l}\text { PERIMETRE } \\
\text { thoracique }\end{array}$ & $\begin{array}{l}\text { PERIMETRE } \\
\text { abdominal }\end{array}$ & $\begin{array}{c}\text { POIDS } \\
\text { au } \\
\text { pont-bascule }\end{array}$ & $\begin{array}{l}\text { PRODUIT } \\
T \times A \times H\end{array}$ & $\begin{array}{l}\text { RAPPOR'I } \\
\text { P TAH }\end{array}$ \\
\hline \multirow[t]{14}{*}{ (I) } & (2) & (3) & (4) & (5) & (6) & (7) & (8) & (9) & (10) & (11) \\
\hline & 1 & 2 & $\mathrm{H}$ & 6 & 1,85 & 2,11 & 2,39 & 460 & 9,3293 & 49,84 \\
\hline & 2 & 371 & $\mathrm{H}$ & 5 & 1,78 & 1,81 & 1,92 & 340 & 6,1858 & 54,96 \\
\hline & 3 & 134 & $E$ & 7 & 1,78 & 2,04 & 2,21 & 400 & 8,0249 & 49,84 \\
\hline & 4 & 212 & E & 8 & 1,80 & 2,02 & 2,19 & 450 & 7,9628 & 56,51 \\
\hline & 5 & 1325 & $\mathrm{H}$ & 5 & 1,81 & 1,94 & 2,08 & 390 & 7,3037 & 52,39 \\
\hline & 6 & 516 & $E$ & 4 & 1,80 & 1,94 & 2,10 & $390 !$ & 7,3332 & 53,04 \\
\hline & 7 & 502 & $\mathrm{E}$ & 6 & 1,85 & 2,09 & 2,20 & 440 & 8,5063 & 51,72 \\
\hline & 8 & 491 & $E$ & 8 & 1,88 & 2,01 . & 2,33 & 455 & 8,8046 & 58,67 \\
\hline & 9 & 525 & $E$ & 6 & 1,75 & 1,91 & 2,13 & 380 & 7,1195 & 53,33 \\
\hline & 10 & 1326 & $\mathrm{H}$ & 6 & 1,78 & 1,88 & 2,06 & 390 & 6,8935 & 56,57 \\
\hline & 11 & 412 & $\mathrm{H}$ & 6 & 1,81 & 1,89 & 1,90 & 340 & 6,4997 & 52,30 \\
\hline & 12 & 515 & $E$ & 5 & 1,82 & 1,92 & 1,92 & 350 & 6,7092 & 52,16 \\
\hline & 13 & 193 & $E$ & 5 & 1,76 & 1,91 & 2,10 & 360 & 7,0593 & 50,99 \\
\hline
\end{tabular}

Valeur de cette relation. - Il restait à verifier la valeur de cette relation. 35 montures des Goums de Kenadza, Taghit et Béchar permirent de le faire (27 et 29 mars 1945). Dans le tableau II' sont consignés les résultats; dans la colonne 9 , nous avons les poids donnés par le pont-bascule; dans la colonne 10, ceux obtenus par la formule $\mathrm{P}=53 \mathrm{TAH}$, l'approximation apparait dans la colonne 11. (Voir ci-contre)

A la lumière de ce tableau, on constate que sur 38 résultats il n'y a que 3 approximations qui dépassent 25 kilos. L'une est de 27,60 (chameau no 39), l'autre de 33,52 (chameau 24) et la troisième de 40,55 (chameau 26). L'approximation moyenne est de 13.
Cette vérification n'avait été effectuée qu'à partir de mâles adultes. Sur notre demande, un caid nous envoyait 3 chamelles gravides et 2 chamelons: c'était peu, mais l'inertie des autochtones est si grande. Les résultats sont au tableau III.

La relation $P=53$ TAH se trouve également ici vérifiée. Aucune approximation ne dépasse 25 kilos.

\section{Calcul dn poids net.}

Donc pour obtenir le poids net d'un chameau, il suffit de faire les opérations suivantes :

Périmetre thoracique $(T) \times$ perimetre abdominal $(A) \times$ hauteur $(\mathrm{H}) \times 53$ d'où la formule $\mathrm{P}=53 \mathrm{TAH}$;

TABLEAU III

\begin{tabular}{|c|c|c|c|c|c|c|c|c|}
\hline $\begin{array}{l}\text { NUMÉRo } \\
\text { d'ordre }\end{array}$ & SEXE & AGE & TAMLLE & $\begin{array}{l}\text { PERRMMETRE } \\
\text { thoracique }\end{array}$ & $\begin{array}{c}\text { PERIMETRE } \\
\text { abdominal }\end{array}$ & $\begin{array}{c}\text { POIDS } \\
\text { au } \\
\text { pont-bascule }\end{array}$ & $\begin{array}{l}\text { POIDS } \\
\text { par la relation } \\
53 ; \text { TAH }\end{array}$ & $\begin{array}{l}\text { APPROXI- } \\
\text { MATION }\end{array}$ \\
\hline 52 & $f$ & 7 & 1,65 & 1,85 & 2,07 & 350 & 334,88 & $-15,12$ \\
\hline 53 & $f$ & 7 & 1,66 & 1,82 & 2,07 & 345 & 331,45 & $-13,55$ \\
\hline 54 & q & 7 & 1,68 & 1,90 & 2,08 & 365 & 351,88 & $-13,12$ \\
\hline 55 & $\sigma^{\prime}$ & 1 & 1,37 & 1,36 & 1,59 & 172 & 157,81 & $-14,99$ \\
\hline 56 & $\sigma$ & I & 1,37 & 1,42 & 1,72 & 165 & 177,34 & $+12,34$ \\
\hline
\end{tabular}


les mesures prises en mètres, le poids est obtenu en kilogrammes.

\section{Calcul du poids brut.}

Il est équivalent au poids net si l'animal était à jeun depuis 48 heures. Si l'animal est nourri normaement, le poids brut est le poids net plus la masse alimentaire, contenue, c'est-à-dire celle absorbée noins celle évacuée. Avec un peu d'habilude, cetle masse alimentaire. s'évalue approximativement.

Considérons les animaux du détachement de Béchar, ils ont été gardés dans la carrière du Goum; abreuvés quotidiennement, il leur a été distribué du grain et de la paille. Nous évaluons la masse alimentaire à 40 kilos. Comparons les résultats du tableau IV. On a :

colonne 3 , le poids brut donné par le pontbasçule;

colonne 4 , le poids brut donné par la somme : poids net +40 kilos;

colonne 5, l'approximation.

'Une approximation au-dessus de 25 kilos : cha-. meau no $47: 28,71$.

En conclusion, le comportement bien particulier du dromadaire devant des possibilités alimentaires variables nous fait considérer chez cet animal deux poids vifs : le poids net et le poids brut. Le poids net sera obtenu en partant de certaines mensurations selon la formule $\mathrm{P}=53 \mathrm{TAH}$ avec une approximation négligeable. Le poids brut sera évalué en partant du poids net.

TABLFAU IV

\begin{tabular}{|c|c|c|c|c|}
\hline 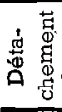 & 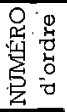 & $\begin{array}{c}\text { POIDS } \\
\text { pont-bascule }\end{array}$ & $\begin{array}{l}\text { POIDS BRUT } \\
53 \mathrm{TAH}+40 \mathrm{~kg}\end{array}$ & $\begin{array}{l}\text { APPROXI- } \\
\text { MATION }\end{array}$ \\
\hline \multirow[t]{5}{*}{ (1) } & (2) & 3 & (4) & (5) \\
\hline & 35 & 435 & 449,34 & $+14,34$ \\
\hline & 36 & 485 & 475,64 & $\therefore 9,36$ \\
\hline & 37 & $475^{\circ}$ & 497,93 & $+22,93$ \\
\hline & 38 & 490 & 474,41 & $-15,59$ \\
\hline \multirow{10}{*}{ 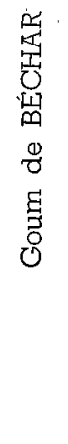 } & 39 & 480 & 467,40 & $-12,60$ \\
\hline & 40 & $485^{\circ}$ & 473,54 & $-11,46$ \\
\hline & 41 & 390. & 404,54 & $+14,54$ \\
\hline & 42 & 515 & 520,37 & $+5,37$ \\
\hline & 43 & 585 & 563,20 & $-21,80$ \\
\hline & 44 & 445 & 445,39 & $+\quad 0,39$ \\
\hline & 45 & 395 & 395,68 & $+0,68$ \\
\hline & 46 & 435 & 457,17 & $+22,17$ \\
\hline & 47 & 425 & 453,71 & $+28,71$. \\
\hline & 48 & $430^{\prime}$ & 439,92 & $+9,92$ \\
\hline
\end{tabular}

